\title{
Una propuesta matemágica para la gamificación en la enseñanza de las matemáticas
}

\author{
A Mathmagical Proposal for Gamification in the Teaching of \\ Mathematics
}

\section{DAVID ALMORZA}

Universidad de Cádiz

(D) http://orcid.org/0000-0002-2004-2799

\section{RESUMEN}

La matemagia, desde hace un tiempo, y la gamificación, de manera más reciente, se han introducido en la docencia de las matemáticas a distintos niveles educativos. Se han desarrollado experiencias docentes con éxito. En estas experiencias se utilizan como herramientas juegos matemáticos con un objetivo docente específico. Sin embargo, en ocasiones son necesarias nuevas herramientas que aporten alguna variedad. En este artículo se presenta un juego de magia con cartas y dados explicado con detalle y con algunas variaciones, a las que se puede orientar la imaginación del alumnado, y que servirán para completar las herramientas utilizadas habitualmente en la docencia.

\section{ABSTRACT}

Mathmagic, for a while, and gamification, more recently, have been introduced in the teaching of mathematics at different educational levels. Successfully teaching experiences have been developed. In these experiences, mathematical games with a specific objective are used as a tool. However, sometimes new tools that provide are variety are necessary. In this paper is presented one magic card and dice game explained in detail and with several variations, to which the student's imagination can be oriented, that can be useful to complete those tools habitually used in teaching.

Recibido: 21/05/20

Aceptado: $11 / 11 / 20$

\section{PALABRAS CLAVES}

Matemáticas; Matemagia; Gamificación; Didáctica de las Matemáticas

\section{KEYWORDS}

Mathematics; Mathmagic; Gamification; Mathematics Education 


\section{INTRODUCCIÓN}

La introducción de juegos para la enseñanza de las matemáticas no es una iniciativa reciente. Se atribuye a Martin Gardner como la persona que empezó a utilizar el término matemagia. Una de las reflexiones de Gardner era: "El mejor método para mantener despierto a un estudiante es seguramente proponerle un juego matemático intrigante, un pasatiempo, un truco mágico, una chanza, una paradoja, un trabalenguas o cualquiera de esas mil cosas que los profesores aburridos suelen rehuir porque piensan que son frivolidades” (Alegría, 2011).

Cuando los juegos seleccionados para la enseñanza de las matemáticas eran juegos de magia, el resultado fue que enseguida captaban la atención del alumnado, y lo hacían de una manera especial. Hoy se reconoce a la matemagia como una herramienta útil en la enseñanza de las matemáticas.

Desde hace relativamente poco tiempo se incorporó la gamificación como herramienta educativa. El término gamificación fue incorporado por Nick Pelling en el año 2002 pero su utilización no se extendió hasta el año 2010, tal como recogen Kamasheva, Valeev, Yagudin y Maksimova (2015).

La gamificación consiste en aplicar dinámicas típicas de los juegos en situaciones no recreativas para cambiar o potenciar la motivación y la respuesta de los individuos ante la consecución de determinados objetivos (Muñoz, Hans y Fernández-Aliseda, 2019).

Holguín, Vila, Baldeón y Chávez (2018) realizaron un experimento con estudiantes de seis y siete años de edad para la enseñanza del conteo y la numeración, y obtuvieron diferencias estadísticamente significativas cuando usaron la gamificación para la enseñanza, logrando un mejor aprendizaje que con otros métodos.

Estos resultados también se logran utilizando matemagia. Fernández y Lahiguera (2015) realizan un trabajo con un total de 555 estudiantes de segundo ciclo de Educación Primaria, todos pertenecientes a un entorno rural. Entre sus conclusiones destaca que "combinando el juego, la diversión y la ilusión con los contenidos matemáticos, se logra captar la atención de los estudiantes y se consigue que quieran aprender más”.

Ni gamificación ni matemagia se limitan a los niveles educativos iniciales, ni tampoco a la docencia de las matemáticas. A nivel universitario Marín, Montejo y Campaña (2016) exponen su experiencia usando gamificación con la plataforma Kahoot! para explicar matemáticas a estudiantes del grado en Administración y Dirección de Empresas, y por su parte Aguado (2017), aplica ideas de la matemagia en la docencia de la Economía.

La plataforma Kahoot! es solo una más de las que se emplean en gamificación. Los softwares gamificados se encuentran ampliamente extendidos, y se han mostrado evidencias sobre su efecto positivo sobre el rendimiento de los estudiantes en el aprendizaje de las matemáticas (Holguín, Holguín y García, 2020).

Sin embargo, este tipo de software no siempre resulta sencillo de encontrar. Las condiciones de búsqueda no son fáciles: que sean gratuitos, que estén disponibles en un idioma asequible para los niños, que se localicen en sitios seguros de Internet, que sean legales y que respeten los derechos de autor... En un reciente trabajo Fernández (2020) propone una serie de lugares que pueden ser de utilidad para el profesorado en este sentido.

Pero tanto, gamificación y matemagia se pueden complementar. Un juego de cartas, por ejemplo, no deja de ser un juego y entraría dentro de la categoría de gamificación. A su vez, un juego de magia con cartas es un proceso en el que hay que seguir ciertas normas. A continuación, el juego que se propone es descubrir, con las orientaciones necesarias, por qué funciona y, si es posible, que el alumnado pueda inventar nuevos juegos a partir de ese. 
Llega un momento en el que estos juegos de magia, estas herramientas de la docencia, escasean, y hay que recurrir a trabajos más especializados y específicos que expliquen y desarrollen un juego para que luego pueda ser trasladado a las aulas. Es el caso, por ejemplo, de González-Puelles y Fragueiro (2018) que presentan el trabajo que realizaron con una batería de juegos matemáticos enfocados a estudiantes de Educación Primaria.

En este punto es donde incluimos una aportación que combina dados y cartas. A continuación, se realizará la presentación y descripción detallada de este juego que es de los llamados automáticos o matemáticos, que son los que no necesitan de ningún tipo de engaño o manejo especial para que resulten (Fernández y Lahiguera, 2015). De esta forma podrán ser utilizados por el profesorado responsable en las aulas donde se estime conveniente utilizarlos. Quizás fuera apropiado para alumnado de tercer ciclo de Educación Primaria.

\section{EL MISTERIO DEL 5 DE CORAZONES}

Como todos los juegos de cartas clásicos, el nombre del juego va cambiando según el mago que los realice, el efecto especial que se quiera dar o la historia con que se ponga en escena. Este mismo juego, con el nombre de "¿existe el azar?", lo presenta de una manera excelente el mago Gustavo Otero. En este caso hemos elegido la carta 5 de corazones como efecto final y esa es la razón del nombre que se ha dado. Añadir que todas las variaciones del juego que se incluyen son de elaboración propia.

En este juego el mago hace la predicción de que al final del conteo aparecerá una carta igual a la que tiene (visible o no) sobre la mesa y que en este caso es el 5 de corazones. El procedimiento implica dados y cartas a la vez, en un procedimiento participativo que al final tiene un efecto visual que atraerá la atención del alumnado.

\subsection{Descripción del juego}

Se utilizan cuatro dados con las caras numeradas del uno al seis, que los espectadores pueden lanzar y comprobar que no están trucados para el juego, es decir, deben comprobar que no se trata de dados falsos. Cuando así lo decidan lanzarán sus dados y obtendrán cuatro resultados (iguales o distintos) que se dejarán en una fila sobre la mesa. El mago sostiene mientras tanto una baraja francesa en sus manos.

A continuación, el mago extraerá de la baraja y colocará junto a cada dado tantas cartas como falten hasta llegar a seis, contando a partir del número que indica el dado, es decir, incluyéndolo. Por ejemplo, si el número es un cinco, el mago pondrá dos cartas a su lado, una por el cinco y otra por el seis. Así todos los dados tendrán por lo menos una carta a su lado.

Para terminar, sumará los números que indican los dados y pondrá sobre la mesa una a una, tantas cartas como indique el resultado de la suma anterior. La última carta que se muestre coincidirá con la predicción del mago y será, como indica el nombre del juego, el 5 de corazones (Imagen 1). 
Imagen 1

Dados, cartas y efecto del juego.

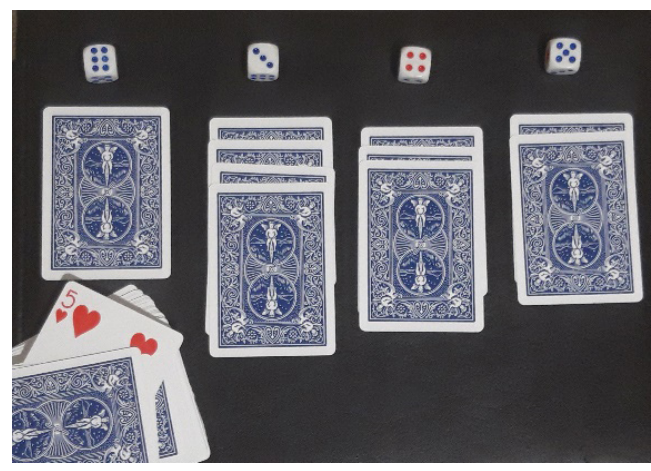

\section{2. ¿Qué aprenderemos con este juego?}

Se trabajará la asignación de letras a los números que no conozcamos, como se verá en el desarrollo, pudiendo servir para introducir la idea de que ese número desconocido y que se quiere calcular es una incógnita. También se fomenta la creatividad, al permitir que el alumnado plantee nuevos juegos derivados de éste; la lógica, que le permitirá discernir entre variantes que se puedan realizar; y la imaginación, porque es la que impregna el desarrollo. Además, en la fase final se recuerda la tabla de multiplicar del siete.

\subsection{Explicación del juego}

Se trata de un juego de los que se conocen como automático o matemático. Necesita una preparación previa, y es que el mago debe tener colocada la carta que le interesa, el 5 de corazones, en la posición veintiocho de la baraja contada a partir de la parte superior. No es necesario nada más.

El juego se puede hacer más visual si se simula algún tipo de mezcla con las cartas de la baraja, pero si va a suponer alguna complicación para el mago no es necesario hacerla. Se puede utilizar una baraja española, francesa o de otro tipo. Lo único que hay que tener en cuenta es colocar la predicción en la posición veintiocho.

\subsection{Justificación del juego.}

El menor resultado que pueden proporcionar los dados es que todos repitan el número 1, es decir: 1-1-1-1. Como en el efecto se repiten cartas incluido ese número, el número de cartas que se pondrá sobre la mesa es de 24 . La suma de los resultados de los dados es 4 , por lo que la posición de la predicción se localizará en la cuarta carta. De esta forma: $24+4=28$.

Pensando del mismo modo, el mayor resultado que pueden proporcionar los dados es que todos repitan el número 6, es decir: 6-6-6-6. Como en el efecto se repiten cartas incluido ese número, el número de cartas que se pondrá sobre la mesa es 4 . La suma de los resultados de los dados es 24, por lo que la posición de la predicción se localizará en la cuarta carta. De esta forma: $4+24=28$. 
Ahora es un buen momento para orientar al alumnado en usar letras para indicar números. Supongamos que no sabemos los resultados de los dados y que les vamos a llamar $a, b, c, y d$. La suma de estos resultados, que nos hace falta para el juego será: $a+b+c+d$.

Junto a cada dado tendremos un montón de cartas en el que habrá como mínimo una sola carta, si el resultado del dado es un 6, o seis cartas como máximo, si el resultado del dado es un 1. Es decir, si se suma el número de cartas de cada montón más el número que aparece en el dado, el resultado es 7 . El número de cartas de cada montón será por tanto $7-a, 7-b, 7-c$ y $7-d$. El número total de cartas sobre la mesa será $28-(a+b+c+d)$.

Si buscamos ahora en la baraja la posición que indica la suma de los dados, se llegará a la posición número 28 ya que lo que estamos haciendo es: $28-(a+b+c+d)+(a+b+c+d)=28$. Con lo que cualquiera que sea la combinación del resultado de los cuatro dados, la posición de la carta siempre se localiza en el número veintiocho.

\subsection{Variaciones del juego}

Una vez que el juego ha sido entendido por parte del alumnado, incluyendo su justificación, se pueden preguntar sobre algunas variaciones del mismo. De esta forma el alumnado entenderá que se puede ir un poco más allá y descubrir que también pueden inventar un nuevo juego. A continuación, se proponen cinco preguntas que harán pensar y permitirán descubrir variantes y limitaciones del juego.

\subsection{1 ¿Se podrían utilizar tres dados?}

Si se utilizan tres dados hay que considerar que la posición inicial de la carta ya no sería el lugar 28, sino el lugar 21. El número de cartas que se van a contar vendrá determinado por el máximo resultado que se puede obtener al sumar tres dados (18), al que hay que añadir 3, porque se añadirán cartas contando a partir del número obtenido con cada dado inclusive.

\subsection{2. ¿Se podrían utilizar dos dados?}

Por el mismo razonamiento anterior, si se utilizan dos dados hay que considerar que la posición inicial de la carta utilizada en la predicción será el lugar 14.

\subsection{3 ¿Se podría utilizar solo un dado?}

Por el mismo razonamiento, si se utiliza solo un dado habría que considerar que la posición inicial de la carta será el lugar 7. Cuando se va disminuyendo el número de dados, el efecto mágico pierde atractivo.

\subsubsection{Entonces, ¿se podrían utilizar cinco dados?}

Podrían emplearse cinco dados, pero entonces la posición inicial de la carta debe ser el lugar 35. Al aumentar el número de dados la posición inicial de la carta se acerca hacia el final de la baraja. 


\subsection{5 ¿Hasta cuántos dados se podrían utilizar?}

Como se puede observar, se está siguiendo la tabla de multiplicar del siete. La posición inicial de la carta se desplaza al lugar que se obtenga de multiplicar por siete el número de dados que se utilicen. Si se realiza el juego con seis dados, la posición de la carta debe ser la 42, y con una baraja española de cuarenta cartas, no se podría realizar. Sin embargo, de utilizar una baraja francesa de 52 cartas, podría hacerse el juego hasta con siete dados, porque la posición sería la 49. También hay que considerar que al aumentar el número de cartas, el conteo se alarga y el espectador puede aburrirse.

\subsection{6 ¿Cómo adaptar el juego para seguir la tabla de multiplicar del seis?}

A estas alturas se ha llegado a una profundidad en el conocimiento del juego que permitiría adaptarlo a seguir la tabla de multiplicar del seis. Tan solo habría que cambiar el procedimiento inicial que se ha descrito, y en vez de disponer tantas cartas al lado de cada dado como números faltan hasta llegar a seis repitiendo el valor que aparece en el dado, hacer el mismo procedimiento sin repetir el número que aparezca en el dado.

Por ejemplo, si el número es un cinco, el mago pondrá solo una carta a su lado, porque no se repite ese valor y solo habría falta una carta para llegar a seis. En consecuencia, podría ocurrir que hubiera algún dado sin carta a su lado, precisamente cuando salga el número seis en el dado. No debemos olvidar que, al hacerlo de esta manera, la posición de la carta de referencia, el 5 de corazones, ya no estará en el lugar veintiocho, sino que habrá bajado a la posición veinticuatro.

\section{Reflexiones finales}

Parece un juego sencillo, pero tiene un gran potencial. En matemagia no se trata de que el alumnado se quede en la sorpresa del juego, sino en que aprenda a conocerlo y pueda plantear, o al menos pensar, en variaciones sobre el mismo. En ese momento está jugando a cambiar el juego. Es el proceso de gamificación que se asocia a la matemagia.

En el apartado de Variaciones del Juego (2.5) se presentan seis variaciones que han sido preparadas expresamente para este artículo, y en las que cada una de ellas constituye por sí misma un nuevo juego de magia. Se han presentado en forma de preguntas, para que puedan servir al profesorado en su labor de guía hacia nuevos descubrimientos, que aquí son juegos, de su alumnado.

\section{Referencias}

Aguado, J.C. (2017). El uso de la magia como recurso docente: el taller de la magia de la economía. Teaching and Learning Innovation Journal, 1, 9 - 13.

Alegría, P. (2011). Magia y Matemagia de la Mano de Martin. Números - Revista Didáctica de las Matemáticas, 76, $19-29$. 
Fernández, M.C. (2020). Recursos educativos abiertos: jugando y aprendiendo matemáticas. Entretextos, 10 (30), $55-67$.

Fernández, R. y Lahiguera, F.J. (2015). Matemagia y su influencia en la actitud hacia las matemáticas en la escuela rural. Números - Revista Didáctica de las Matemáticas, 89, 33 - 53.

González-Puelles, I. y Fragueiro, M.S. (2018). Una experiencia de aula basada en los juegos de magia como herramienta pedagógica en educación primaria. EA, Escuela Abierta, 21, 77 - 93.

Holguín, J.A., Vila, G.M., Baldeón, M.D. y Chávez, Y. (2018). Didáctica semiótica y gamificación matemática no digital en niños de un Complejo Municipal Asistencia Infantil. Fides Et Ratio, 16 (Sep.), 147 - 168.

Holguín, F.Y., Holguín, E.G. y García, N.A. (2020). Gamificación de la enseñanza de las matemáticas: una revisión sistemática. Telos: revista de Estudios Interdisciplinarios en Ciencias Sociales, 22 (1), 62 - 75.

Kamasheva A.V., Valeev, E.R., Yagudin, R. Kh. y Maksimova, K.R. (2015). Usage of Gamification Theory for Increase Motivation of Employees. Mediterranean Journal of Social Sciences, 6 (1), 77 - 80.

Marín, A.E., Montejo, J. y Campaña, J.R. (2016). Una propuesta para el refuerzo de conceptos matemáticos a través de Kahoot! Revista CIDUI 2016, 3. Recuperado el 21/05/2020 de https://www.cidui.org/revistacidui/index. $\mathrm{php} /$ cidui/article/view/1060

Muñoz, J., Hans, J.A. y Fernández-Aliseda, A. (2019). Gamificación en matemáticas, ¿un nuevo enfoque o una nueva palabra? Épsilon - Revista de Educación Matemática, 101, 29 - 45.

\section{INFORMACIÓN SOBRE EL AUTOR}

David Almorza. Doctor en Ciencias Matemáticas por la Universidad de Cádiz. Profesor Titular de Universidad en el Departamento de Estadística e Investigación Operativa. Adscrito a la Facultad de Ciencias del Trabajo de la Universidad de Cádiz. Una de sus líneas de investigación incluye el campo de la educación. 